\title{
MULTIDIMENSIONAL CREDIBILITY MODEL AND ITS APPLICATION
}

\author{
Viera Pacáková, Erik Šoltés, Bohdan Linda
}

\section{Introduction}

A huge expansion of a competitive insurance market forms part of the transition process in the former Socialist countries. New international trends in securing its financial stability and new types of risk demand a high-powered development of actuarial science and its application in insurance practice. The on-going European Union Solvency II project, aimed at improving the quality of assessment of the solvency of an insurer, places emphasis on the modelling of risk and on internal models for managing an insurer's risk. This unavoidably leads to a more in depth use of the theoretical results of actuarial science and to their further theoretical development.

Improving the quality of premium calculation methods is an effective factor in reducing the insurance technical risk of an insurer. In doing so it takes into account more and more rating factors, thanks to which more homogeneous rating tariffs emerge allowing the possibility of determining more equitable premiums. On the other hand when the insurer determines the premium it has to contend with a smaller amount of information concerning the risk, for which the given tariff class is intended. If the insurer has inadequate past data relating to the tariff class, it can make use of data from other risk classes which are to a given extent similar.

The insurance risk, for which there is a given tariff class, is called the individual risk. Each tariff class forms part of the whole portfolio and thus each individual risk is part of the collective, i.e. portfolio, risk. On the one hand the insurer in general has at its disposal a relatively large amount of statistical information concerning the collective risk. This information is, however, more or less limited for pricing individual risks, due to the wide variety of tariff classes. An insurer usually has little statistical information concerning individual risks and therefore should use both sources of data. That is the fundamental idea behind credibility theory.

Back in 1918 Albert W. Whitney proposed that the credible premium Pcred should be a linear combination of the individual premium $P$ ind and the collective premium Pcol

$$
\text { Pcred }=z . \text { Pind }+(1-z) \cdot \text { Pcol }
$$

where Pind is the average level of claim per contract in the given tariff class and $P c o l$ is the respective mean for the whole insurance portfolio. From a statistical point of view the credible premium is the weighted arithmetical average of the individual and collective premiums, where $z$ is the relative weight of the individual premium and $(1-z)$ that of the collective premium. The weight $z$ is referred to as the credibility factor. It takes values in the interval $\langle 0 ; 1\rangle$ and expresses the level of confidence in the data for the given insurance class when determining the premium for that class. A confidence level (weight) of 0 is assigned to individual risk data which have no use for determining the premium and a level of 1 is given to those individual risk data which completely suffice in order to determine the premium for that risk class. This means that, if $z=0$, we must completely rely on the data from comparable risks when determining the premium for the given class. If $z=1$, then we can completely rely on the data for the given individual risk.

Bühlmann established the theoretical foundation of modern credibility theory, presented as distribution free credibility estimation. Bühlmann and Straub (see [3]) generalized Bühlmann's classical credibility model. Without 
doubt the Bühlmann-Straub model is the most important model in credibility theory. Originally it was aimed at the determination of the credible premium, but after certain modifications it can also be used to determine a reliable estimate of claims frequency or of the average amount of claim. Its importance lies in the possibility of using it in a wide range of applications in the actuarial practice of non-life insurance, but it can also be used in life insurance and in reinsurance. Besides that the Bühlmann-Straub model represents a base for further more specific models, such as the hierarchical, multidimensional or regression credibility models. In this paper we will consider a generalisation of the one-dimensional Bühlmann-Straub credibility model to the multidimensional credibility model.

\section{Generalisation of the One- Dimensional Bühlmann-Straub Model to the Multidimensional Credibility Model}

The aim of this part of article is to briefly describe the mathematical and statistical basis for one-dimensional Bühlmann-Straub model (for details see [1] to [7]) and point out the analogy of multidimensional Bühlmann-Straub model with the original Bühlmann-Straub model. This part of the article also shows the practical use of multi-dimensional BühlmannStraub model in insurance practice.

Let us suppose that the portfolio of contracts is divided into / risk classes, for which we want to determine the net premium. Let the th risk class $(i=1,2, \ldots, l)$ be characterised by the individual risk profile $\vartheta_{i}$, which is the outcome of a random variable $\theta_{i}$. To calculate the net premium for each individual risk class we will make use of the quantities:

$S_{i j}$ - the aggregate claim amount for risk $i$ in year $j(j=1,2, \ldots, n)$, year $j$

$w_{i j}$ - the number of contracts for risk $i$ in

$$
X_{i j}=\frac{S_{i j}}{w_{i j}}-\text { the average annual level of }
$$

claim for risk $i$ in year $j$, which are in general known.

The one dimensional Bühlmann-Straub credibility model stems from the following assumptions Bühlmann, Straub [3], Herzog [6], Pacáková [8].

$B S_{1}$ : Conditional on the value of $\theta_{i}$ the $X_{i j}$ for $j=1,2, \ldots, n$ are independent with parameters:

$$
\begin{aligned}
& E\left[X_{i j} \mid \theta_{i}\right]=\mu\left(\theta_{i}\right) \\
& \operatorname{Var}\left[X_{i j} \mid \theta_{i}\right]=\frac{\sigma^{2}\left(\theta_{i}\right)}{w_{i j}}
\end{aligned}
$$

$B S_{2}$ : The pairs $\left(\theta_{1}, X_{1}\right),\left(\theta_{2}, X_{2}\right) \ldots$ are independent and $\theta_{1}, \theta_{2}$ are independent and identically distributed.

If the assumptions $B S_{1}$ a $B S_{2}$ are met, the credible estimate of the net premium is

$$
{\widehat{{\widehat{\mu\left(\theta_{i}\right)}}^{B-S}}}^{B-} z_{i} \cdot \bar{X}_{i}+\left(1-z_{i}\right) \cdot \mu_{0}
$$

where the average annual level of claim per contract from the ith risk class

$$
\overline{X_{i}}=\sum_{j=1}^{n} X_{i j} \frac{w_{i j}}{w_{i \bullet}} \quad w_{i \bullet}=\sum_{j=1}^{n} w_{i j}
$$

is the estimate of the individual premium, $\mu_{0}$ is the collective premium and the credibility factor has the form

$$
z_{i}=\frac{w_{i \bullet}}{w_{i \bullet}+\frac{\sigma^{2}}{\tau^{2}}}=\frac{\tau^{2}}{\tau^{2}+\frac{\sigma^{2}}{w_{i \bullet}}}
$$

The estimation of the so-called structural parameters $\mu_{0}, \sigma^{2}$ a $\tau^{2}$ plays a fundamental role in credibility theory. Definitions of the structural parameters and their interpretation are as follows:

Structural parameter

$\mu_{0}=E\left[\mu\left(\theta_{i}\right)\right]$

$\sigma^{2}=E\left[\sigma^{2}\left(\theta_{i}\right)\right]$, $\sigma^{2}\left(\theta_{i}\right)=\operatorname{Var}\left[X_{i j} \mid \theta_{i}\right] \cdot w_{i j}$ $\tau^{2}=\operatorname{Var}\left[\mu\left(\theta_{i}\right)\right]$, where $\mu\left(\theta_{i}\right)=E\left[X_{i j} \mid \theta_{i}\right]$

The credibility estimate (4), in which we replace the unknown collective premium $\mu_{0}$ by the estimate 


$$
\widehat{\widehat{\mu_{0}}}=\sum_{i=1}^{I} \overline{X_{i}} \frac{z_{i}}{z_{\bullet}}, \quad z_{\bullet}=\sum_{i=1}^{I} z_{i}
$$

is the so-called homogeneous credibility estimate with the form (see [4])

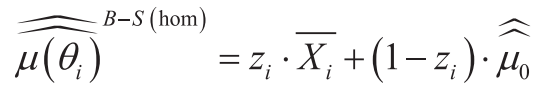

The estimate (7) of the collective premiumis the weighted arithmetical average of the individual premiums $\bar{X}_{j}$, where the weights are the credibility factors $z_{i}$.

Assuming that there is the same number of contracts in each risk class in each year, the credible premium defined by formula (8) should cover the claims outgo for the whole portfolio and for the whole period of $\mathrm{n}$ previous years considered. This result is expressed in [1] by the balance property:

$$
\sum_{j=1}^{n} \sum_{i=1}^{I}\left[{\widehat{\widehat{\mu\left(\theta_{i}\right)}}}^{B-S(\mathrm{hom})} \cdot w_{i j}\right]=\sum_{j=1}^{n} \sum_{i=1}^{I}\left[X_{i j} \cdot w_{i j}\right] \text { (9) }
$$

For some classes of business (liability, commercial fire) a high percentage of the total claim amount comes from a small number of very large claims. In these cases a relatively small number of claims, which are "large" compared with the "standard" claim, have a large weight when the premium rate is determined. It therefore makes sense to estimate the average claim amount separately for "large" and for "standard" claims. If in fact we were to calculate the credible premium using the one-dimensional Bühlmann-Straub model separately for "large" and "standard" claims, we would not take into account any dependency between them. Therefore it is more appropriate to estimate the vector of net premiums, which in this case is the two-dimensional vector

$$
\boldsymbol{\mu}^{\mathrm{T}}\left(\theta_{i}\right)=\left(\mu_{1}\left(\theta_{i}\right) \quad \mu_{2}\left(\theta_{i}\right)\right) .
$$

The whole vector of net premiums for individual risk i can be estimated by the multidimensional credibility model, which is a generalisation of the one-dimensional Bühlmann-Straub model.

Another example of the use of the multidimensional model would be the calculation of the credible premium for the separate risk classes of a whole portfolio and the similar risk classes of other insurers. If, therefore, an insurer disposes of data, even from another insurer, either broken-down or in total, it can use the multidimensional credibility model. Thus, thanks to it, we can calculate the premium for a given risk class using either past data for that class or past data for the whole of the portfolio or even historical data for the given risk class or for the whole portfolio of similar risks of another insurer.

Whereas in the one-dimensional Bühlmann-Straub credibility model we estimate the net premium $\mu\left(\theta_{j}\right)$ for the risk $i(i=1,2, \ldots, l)$, in the multidimensional Bühlmann-Straub credibility model we estimate for risk i the whole vector of net premiums

$$
\boldsymbol{\mu}^{\mathrm{T}}\left(\theta_{i}\right)=\left(\begin{array}{llll}
\mu_{1}\left(\theta_{i}\right) & \mu_{2}\left(\theta_{i}\right) & \ldots & \mu_{p}\left(\theta_{i}\right)
\end{array}\right)
$$

In the one-dimensional Bühlmann-Straub credibility model we observe the quantities $X_{i j}$ (the average annual claim amount for risk $i$ in year $j$ ) with weights $w_{i j}$ (the number of contracts for risk $i$ in year $j$ ). In the multidimensional Bühlmann-Straub credibility model we observe the vector of average annual claim amounts for risk $i$ in year $j$

$$
\mathbf{X}_{i j}^{\mathrm{T}}=\left(\begin{array}{llll}
X_{i j}^{(1)} & X_{i j}^{(2)} & \ldots & X_{i j}^{(p)}
\end{array}\right)
$$

and the weights are the vector of the number of contracts for risk $i$ in year $j$

$$
\mathbf{w}_{i j}^{\mathrm{T}}=\left(\begin{array}{llll}
w_{i j}^{(1)} & w_{i j}^{(2)} & \ldots & w_{i j}^{(p)}
\end{array}\right)
$$

The multidimensional Bühlmann-Straub credibility model stems from the following assumptions (for details see [2]):

$M B S_{1}$ : Conditional on the value of $\theta_{1}$ the values $X_{i j}$ for $j=1,2, \ldots, n$ are independent with a p-dimensional vector of mean values:

$$
E\left[\mathbf{X}_{i j} \mid \theta_{i}\right]=\boldsymbol{\mu}\left(\theta_{i}\right)
$$

and with a covariance matrix of type $p \times p$ 
$\operatorname{Cov}\left(\mathbf{X}_{i j}, \mathbf{X}_{i j}^{\mathrm{T}} \mid \theta_{i}\right)=\left(\begin{array}{cccc}\frac{\sigma_{1}^{2}\left(\theta_{i}\right)}{w_{i j}^{(1)}} & 0 & \cdots & 0 \\ 0 & \frac{\sigma_{2}^{2}\left(\theta_{i}\right)}{w_{i j}^{(2)}} & \cdots & 0 \\ \vdots & \vdots & \ddots & \vdots \\ 0 & 0 & \cdots & \frac{\sigma_{p}^{2}\left(\theta_{i}\right)}{w_{i j}^{(p)}}\end{array}\right)$

$M_{B S}:$ The pairs $\left\{\left(\theta_{i}, \mathbf{X}_{i}\right): i=1,2, \ldots, I\right\}$, where the $\mathbf{X}_{i}^{\mathrm{T}}=\left(\begin{array}{llll}\mathbf{X}_{i 1}^{\mathrm{T}} & \mathbf{X}_{i 2}^{\mathrm{T}} & \ldots & \mathbf{X}_{i n}^{\mathrm{T}}\end{array}\right)$ are independent and the $\theta_{1}, \theta_{2}, \ldots$ are independent and identically distributed.

The covariance matrix (14) is diagonal, thus it is assumed that the components of vector $X_{i j}$ are independent. In some situations independence is not assured. For example, if we want to use the multidimensional credibility model to estimate the claim frequencies of larger claims and the claim frequencies of standard claims, the assumption of their independence is not met. The claim frequencies of large claims and of standard claims relate to the same number of contracts and therefore the weights $w_{i j}^{(1)}$ a $w_{i j}^{(2)}$ are equal. In the case of equality of the weights $w_{i j}^{(1)}=\ldots=w_{i j}^{(p)}=w_{i j}$ we can replace the socalled standard assumption by (see [1]) the socalled alternative assumption:

In assumption $M B S_{1}$ in place of the covariance matrix (14) we can consider the covariance matrix

$$
\operatorname{Cov}\left(\mathbf{X}_{i j}, \mathbf{X}_{i j}^{\mathrm{T}} \mid \theta_{i}\right)=\frac{1}{w_{i j}} \boldsymbol{\Sigma}\left(\theta_{i}\right)
$$

where the weights for all the components of the vector $X_{i j}$ are equal, i.e. $w_{i j}^{(1)}=\ldots=w_{i j}^{(p)}=w_{i j}$

The standard and alternative assumptions do not in fact cover all possible situations, but they do include the majority of cases which arise in practice. Subject to these conditions we can generalise formulae (4) to (6), relating to the one-dimensional credibility estimate, using the multidimensional credibility estimate defined later by formulae (16) to (21).

If assumptions $M B S_{1}$ a $M B S_{2}$ are met, we can express the credibility estimate of the net premium as follows

$$
\widehat{\widehat{\boldsymbol{\mu ( \theta _ { i } )}}}=\mathbf{Z}_{i} \cdot \mathbf{B}_{i}+\left(\mathbf{I}-\mathbf{Z}_{i}\right) \cdot \boldsymbol{\mu}
$$

where $B_{i}$ is the individual estimate and $\mu$ the collective estimate of the vector $\mu\left(\theta_{\mathrm{i}}\right)$. $\mathbf{Z}_{\mathrm{i}}$ is the credibility matrix and it can be shown that, after generalising formula (6) for the credibility factor, the following applies

$$
\mathbf{Z}_{i}=\mathbf{T} \cdot\left(\mathbf{T}+\mathbf{W}_{i \bullet}^{-\frac{1}{2}} \cdot \mathbf{S} \cdot \mathbf{W}_{i \bullet}^{-\frac{1}{2}}\right)^{-1}
$$

It is clear that the vector of average annual claim amounts for the th risk class represents the individual estimate of the vector $\mu\left(\theta_{i}\right)$ and therefore

$$
\mathbf{B}_{i}=\left(\begin{array}{llll}
B_{i}^{(1)} & B_{i}^{(2)} & \ldots & B_{i}^{(p)}
\end{array}\right)^{\mathrm{T}}
$$

here, for $k=1,2, \ldots, p$, the $k^{\text {th }}$ element of the vector $\mathbf{B}_{i}$ is

$$
B_{i}^{(k)}=\sum_{j=1}^{n} X_{i j}^{(k)} \frac{w_{i j}^{(k)}}{w_{i \bullet}^{(1)}}
$$

We can therefore express the vector $\mathbf{B}_{i}$ by the formula

$$
\mathbf{B}_{i}=\mathbf{W}_{i \bullet}^{-1} \cdot \sum_{j=1}^{n}\left(\mathbf{X}_{i j} \cdot \mathbf{W}_{i j}\right)
$$

where the matrix of weights has the elements

$$
\mathbf{W}_{i j}=\left(\begin{array}{cccc}
w_{i j}^{(1)} & 0 & \cdots & 0 \\
0 & w_{i j}^{(2)} & \cdots & 0 \\
\vdots & \vdots & \ddots & \vdots \\
0 & 0 & \cdots & w_{i j}^{(p)}
\end{array}\right)
$$

If standard assumption (14) is met, the matrix $\mathbf{W}_{i \bullet}^{-\frac{1}{2}} \cdot \mathbf{S} \cdot \mathbf{W}_{i \bullet}^{-\frac{1}{2}}$ has elements

$$
\mathbf{W}_{i \bullet}^{-\frac{1}{2}} \cdot \mathbf{S} \cdot \mathbf{W}_{i \bullet}^{-\frac{1}{2}}=\left(\begin{array}{cccc}
\frac{\sigma_{1}^{2}}{w_{i \bullet}^{(1)}} & 0 & \cdots & 0 \\
0 & \frac{\sigma_{2}^{2}}{w_{i \bullet}^{(2)}} & \cdots & 0 \\
\vdots & \vdots & \ddots & \vdots \\
0 & 0 & \cdots & \frac{\sigma_{p}^{2}}{w_{i \bullet}^{(p)}}
\end{array}\right)
$$


If the covariance matrix of the vector meets the alternative assumption (15), the following applies

$$
\mathbf{W}_{i \bullet}^{-\frac{1}{2}} \cdot \mathbf{S} \cdot \mathbf{W}_{i \bullet}^{-\frac{1}{2}}=\frac{1}{w_{i \bullet}} \mathbf{S}
$$

The multidimensional credibility model has three structural parameter vectors $\mu, \mathbf{S}$ and $\mathbf{T}$ which are analogous to the structural parameters $\mu_{0}, \sigma^{2}$ and $\tau^{2}$ of the one-dimensional BühImann-Straub credibility model. Their definitions are as follows:

\section{The matrices of structural parameters}

$\boldsymbol{\mu}=E\left[\boldsymbol{\mu}\left(\theta_{i}\right)\right]$ is the vector of collective premiums

$\mathbf{S}=E\left[\Sigma\left(\theta_{i}\right)\right]$, where $\boldsymbol{\Sigma}\left(\theta_{i}\right)=\operatorname{Cov}\left(\mathbf{X}_{i}, \mathbf{X}_{i}^{\mathrm{T}} \mid \theta_{i}\right)$ is the covariance matrix of the vector $\mathbf{X}_{i}=\left(X_{i 1}, \ldots, X_{i n}\right)^{\mathrm{T}}$ $\mathbf{T}=\operatorname{Cov}\left(\boldsymbol{\mu}\left(\theta_{i}\right), \boldsymbol{\mu}\left(\theta_{i}\right)^{\mathrm{T}}\right) \begin{aligned} & \text { is the covariance matrix of the } \\ & \text { collective premium vector }\end{aligned}$

We obtain the homogeneous credibility estimate ${\widehat{\widehat{\mu\left(\theta_{i}\right)}}}^{\text {hom }}$ if in formula (16) we replace the unknown vector $\mu$ of the collective premiums by its estimate

$$
\hat{\hat{\boldsymbol{\mu}}}=\left(\sum_{i=1}^{I} \mathbf{Z}_{i}\right)^{-1} \cdot \sum_{i=1}^{I}\left(\mathbf{Z}_{i} \cdot \mathbf{B}_{i}\right)
$$

Similar to the one-dimensional BühlmannStraub credibility model, also in the multidimensional model the homogeneous estimate and the individual elements of the vector

${\widehat{\widehat{\mu\left(\theta_{i}\right)}}}^{\text {hom }}$ satisfy the balance property

$$
\sum_{j=1}^{n} \sum_{i=1}^{I}\left[{\widehat{\widehat{\mu_{k}\left(\theta_{i}\right)}}}^{\text {hom }} \cdot w_{i j}^{(k)}\right]=\sum_{j=1}^{n} \sum_{i=1}^{I}\left[X_{i j}^{(k)} \cdot w_{i j}^{(k)}\right]
$$

To compare the accuracy of credibility estimates, collective estimate and individual estimates, there are used quadratic losses. The quadratic loss of the credible premium is $z_{i}$-multiple of the quadratic loss of the individual premium and $\left(1-z_{j}\right)$-multiple of the quadratic loss of the collective premium (see [9]). Since $z_{i}$ and $\left(1-z_{i}\right)$ take values from interval $\langle 0 ; 1\rangle$, so the quadratic loss of the credibility premium is less or at the most equal to quadratic losses of the collective and the individual premium.

\section{Estimation of the Structural Parameters of the One- Dimensional Bühlmann-Straub and the Multidimensional Credibility Models}

In the one-dimensional Bühlmann-Straub credibility model it is necessary to estimate, not only the collective premium as shown in formula (8), but also the structural parameters of variability $\sigma^{2}$ and $\tau^{2}$. The structural parameter $\sigma^{2}$ is the mean of the within group variances $\sigma^{2}\left(\theta_{\mathrm{i}}\right)$. As the estimate of the variance $\sigma^{2}\left(\theta_{\mathrm{i}}\right)$ in the ${ }^{\text {th }}$ risk class we can take the sample variance

$$
\widehat{\sigma^{2}\left(\theta_{i}\right)}=\frac{1}{n-1} \sum_{j=1}^{n}\left(X_{i j}-\overline{X_{i}}\right)^{2} w_{i j}
$$

It is possible to estimate the mean of these variances as the arithmetic mean. As reported by BühImann and Gisler [1] the optimal weights should be proportional to $\left(E\left[\left(\sigma^{2}\left(\theta_{i}\right)-\sigma^{2}\right)^{2}\right]\right)^{-1}$. This expression depends on the moments up to the fourth order. These moments are unknown and their estimation requires further structural parameters, therefore such a procedure is not appropriate. In the special case, where $X_{i j}$ are conditionally on $\theta_{i}$ normally distributed, the above mentioned expression does not depend on $i$. Therefore it is optimal to use the equal weights for all partial variances. On the one hand, the most of insurance data are not normally distributed, on the other hand, weights, which would be better, are not known. For this reason, Bühlmann and Straub [3] suggested to estimate the structural parameter $\sigma^{2}$ using the simple arithmetic mean of the individual variances $\widehat{\sigma^{2}\left(\theta_{i}\right)}$ :

$$
\widehat{\sigma^{2}}=\frac{1}{I} \sum_{i=1}^{I} \widehat{\sigma^{2}\left(\theta_{i}\right)}=\frac{1}{I} \sum_{i=1}^{I} \frac{1}{n-1} \sum_{j=1}^{n}\left(X_{i j}-\overline{X_{i}}\right)^{2} w_{i j}
$$

The characteristic $\widehat{\sigma^{2}}$ is an unbiased and consistent estimate of the structural parameter $\sigma^{2}$. A reader can find proofs of these properties in [1] on the page 94.

An unbiased and, provided there is no dominant risk class in the portfolio, also consistent estimate of the parameter $\tau^{2}$ (see e.g. [7]) is given by 


$$
\widehat{\widehat{\tau^{2}}}=c \cdot\left\{T-\frac{I \cdot \widehat{\sigma^{2}}}{w_{\bullet \cdot}}\right\}
$$

where

$$
\begin{gathered}
c=\frac{I-1}{I}\left\{\sum_{i=1}^{I}\left(1-\frac{w_{i \bullet}}{w_{\bullet \bullet}}\right) \frac{w_{i \bullet}}{w_{\bullet \bullet}}\right\}^{-1} \\
T=\frac{I}{I-1} \sum_{i=1}^{I}\left(\overline{X_{i}}-\bar{X}\right)^{2} \frac{w_{i \bullet}}{w_{\bullet \bullet}}
\end{gathered}
$$

And

$$
\bar{X}=\sum_{i=1}^{I} \overline{X_{i}} \frac{w_{i \bullet}}{w_{\bullet \bullet}}
$$

Remark:
If the situation arises, that $\widehat{\tau^{2}}$ is negative, differences between the risk classes are not demonstrable. In this case we set the parameter $\tau^{2}$ to 0 . The credibility factors (6) then equal 1 and the credibility premiums for all the risk classes are the same and are equal to the average annual claim amount for the whole portfolio (31).

So that the estimate of the structural parameter $\tau^{2}$ can also be used in the situation described in the remark, the estimate of the structural parameter $\tau^{2}$ is derived from the formula

$$
\widehat{\tau^{2}}=\max \left\{\widehat{\tau^{2}} ; 0\right\}
$$

The homogeneous credibility estimate in the multidimensional credibility model already has incorporated in it an estimate of the vector $\mu$ in accordance with formula (24). Hence we will concentrate only on estimation of the matrix of the structural parameters $\mathbf{S}$ and $\mathbf{T}$.

To estimate the diagonal elements of the matrices $\mathbf{S}$ a $\mathbf{T}$ we can use analogous approaches to those in the case of the onedimensional Bühlmann-Straub model. The diagonal elements of the matrix $\mathbf{S}$ are estimated in a similar way to that of the parameter $\sigma^{2}$ in the Bühlmann-Straub model, in accordance with formula (27) thus:
$\widehat{\sigma_{k}^{2}}=\frac{1}{I} \sum_{i=1}^{I} \frac{1}{n-1} \sum_{j=1}^{n}\left(X_{i j}^{(k)}-B_{i}^{(k)}\right)^{2} w_{i j}^{(k)}$

The diagonal elements of the matrix $\mathbf{T}$ again can be estimated similarly to the structural parameter $\tau^{2}$ in the Bühlmann-Straub model. Analogously to formulae (32) and (28) to (31) we then get

$$
\widehat{\tau_{k}^{2}}=\max \left\{\widehat{\widehat{\tau_{k}^{2}}} ; 0\right\}
$$

where

$$
\widehat{\tau_{k}^{2}}=c^{(k)} \cdot\left\{T^{(k)}-\frac{I \cdot \widehat{\sigma_{k}^{2}}}{w_{\bullet \bullet}^{(k)}}\right\}
$$

and

$$
\begin{gathered}
c^{(k)}=\frac{I-1}{I}\left\{\sum_{i=1}^{I}\left(1-\frac{w_{i \bullet}^{(k)}}{w_{\bullet \bullet}^{(k)}}\right) \frac{w_{i \bullet}^{(k)}}{w_{\bullet \bullet}^{(k)}}\right\}^{-1} \\
T^{(k)}=\frac{I}{I-1} \sum_{i=1}^{I}\left(B_{i}^{(k)}-\overline{B^{(k)}}\right)^{2} \frac{w_{i \bullet}^{(k)}}{w_{\bullet \bullet}^{(k)}} \\
\overline{B^{(k)}}=\sum_{i=1}^{I} B_{i}^{(k)} \frac{w_{i \bullet}^{(k)}}{w_{\bullet \bullet}^{(k)}}
\end{gathered}
$$

We will denote the non-diagonal element in the $k^{\text {th }}$ row and the th column of the matrix $\mathbf{T}$ by $\tau_{\mathrm{kl}}$. First we estimate $\tau_{\mathrm{kl}}$ using the weights $w_{i \bullet}^{(k)}$ thus:

$$
{\widehat{\tau_{k l}}}^{*}=c^{(k)} \cdot\left\{\frac{I}{I-1} \sum_{i=1}^{I}\left(B_{i}^{(k)}-\overline{B^{(k)}}\right) \cdot\left(B_{i}^{(l)}-\overline{B^{(l)}}\right) \frac{w_{w^{(k}}^{(k)}}{w_{\bullet \bullet}^{(k)}}\right\}
$$

Similarly we estimate $\tau_{\mathrm{kl}}$ using the weights $w_{i \bullet}^{(l)}$ :

${\widehat{\tau_{k l}}}^{* *}=c^{(l)} \cdot\left\{\frac{I}{I-1} \sum_{i=1}^{I}\left(B_{i}^{(k)}-\overline{B^{(k)}}\right) \cdot\left(B_{i}^{(l)}-\overline{B^{(l)}}\right) \frac{w_{i \bullet}^{(l)}}{w_{\bullet \bullet}^{(l)}}\right\}$ 
where

$$
c^{(l)}=\frac{I-1}{I}\left\{\sum_{i=1}^{I}\left(1-\frac{w_{i \bullet}^{(l)}}{w_{\bullet \bullet}^{(l)}}\right) \frac{w_{i \bullet}^{(l)}}{w_{\bullet \bullet}^{(l)}}\right\}^{-1}
$$

The matrix $\mathbf{T}$ is the covariance matrix, which means that its diagonal elements are the variances $\tau_{\mathrm{k}}^{2}$ and its non-diagonal elements are the covariances $\tau_{\mathrm{kl}}$. By taking the ratio of the covariance $\tau_{\mathrm{kl}}$ and the product of the standard deviations $\tau_{\mathrm{k}} \cdot \tau_{\text {l }}$ we get the correlation coefficient, which takes values in the interval $\langle-1 ; 1\rangle$. On the basis of this it must be the case that $\left|\tau_{\mathrm{k}}\right| \leq \tau_{\mathrm{k}}$. $\tau_{\mathrm{k}}$. If this condition is met, the simple arithmetic mean of the estimates (39) and (40) is an estimate of the covariance $\tau_{\mathrm{kl}}$. In general the following estimate is proposed (see [1])

$$
\widehat{\tau_{k l}}=\operatorname{sgn}\left(\frac{\widehat{\tau_{k l}}+\widehat{\tau_{k l}}}{2}\right) \cdot \min \left(\frac{\left|\widehat{\tau_{k l}^{*}}+\widehat{\tau_{k l}^{*}}\right|}{2} ; \sqrt{\widehat{\tau_{k}^{2}} \cdot \widehat{\tau_{l}^{2}}}\right)
$$

To estimate the structural parameters of the one-dimensional and also the multidimensional Bühlmann-Straub credibility model we can use statistical methods, for which we can find procedures in statistical programming packages. We will concentrate only on the multidimensional credibility model. So the $k^{\text {th }}$ diagonal element (33) of the matrix $\mathbf{S}$ is the simple arithmetic mean of the sample variances

$$
\widehat{\sigma_{k}^{2}}=\frac{1}{I} \sum_{i=1}^{I}{\widehat{\sigma_{i}^{2}}}^{(k)}
$$

where

$$
{\widehat{\sigma_{i}^{2}}}^{(k)}=\frac{1}{n-1} \sum_{j=1}^{n}\left(X_{i j}^{(k)}-\overline{X_{i}^{(k)}}\right)^{2} w_{i j}^{(k)}
$$

in each of the risk groups, relating to the $k^{\text {th }}$ element of the estimated vector

$\boldsymbol{\mu}^{\mathrm{T}}\left(\theta_{i}\right)=\left(\begin{array}{llll}\mu_{1}\left(\theta_{i}\right) & \mu_{2}\left(\theta_{i}\right) & \ldots & \mu_{p}\left(\theta_{i}\right)\end{array}\right)$.

Let us modify the elements of matrix T. We can rewrite the characteristics $T(k)$ in the form

$$
T^{(k)}=\frac{I}{w_{\bullet \bullet}^{(k)}} \cdot s_{B^{(k)}, w_{i \bullet}^{(k)}}^{2}
$$

where $S_{B^{(k)}, w_{i e}^{(k)}}^{2}$ is the sample variance of the variable $B(k)$ with relative weights $w_{i \bullet}^{(k)}$ :

$$
s_{B^{(k)}, w_{i \bullet}^{(k)}}^{2}=\frac{1}{I-1} \sum_{i=1}^{I}\left(B_{i}^{(k)}-\overline{B^{(k)}}\right)^{2} w_{i \bullet}^{(k)}
$$

We then know that in accordance with formula (45) we can express the estimate (35) of the diagonal element $\tau_{\mathrm{k}}^{2}$ of the matrix $\mathbf{T}$ by the formula

$$
\widehat{\tau_{k}^{2}}=c^{(k)} \cdot\left\{\frac{I}{w_{\bullet}^{(k)}} \cdot s_{B^{2}\left(w_{i}(k)\right.}^{2}-\frac{I \cdot \widehat{\sigma_{k}^{2}}}{w_{\bullet \bullet}^{(k)}}\right\}=I \cdot \frac{c^{(k)}}{w_{\bullet \bullet}^{(k)}} \cdot\left(s_{B^{(k)}, w_{* \bullet}^{(k)}}^{2}-\widehat{\sigma_{k}^{2}}\right)
$$

In the case of the estimates (39), (40) of the non-diagonal elements $\tau_{\mathrm{kl}}$ of the matrix $\mathbf{T}$ it is again the case that

$$
\begin{aligned}
& {\widehat{\tau_{k l}}}^{*}=I \cdot \frac{c^{(k)}}{w_{\bullet \bullet}^{(k)}} \cdot \operatorname{cov}_{w_{i \bullet}^{(k)}}\left(B^{(k)}, B^{(l)}\right) \\
& {\widehat{\tau_{k l}}}^{* *}=I \cdot \frac{c^{(l)}}{w_{\bullet \bullet}^{(l)}} \cdot \operatorname{cov}_{w_{i:}^{(l)}}\left(B^{(k)}, B^{(l)}\right)
\end{aligned}
$$

where $\operatorname{cov}_{w_{i}^{(l)}}\left(B^{(k)}, B^{(l)}\right)$ is the sample covariance between variables $B(k), B(l)$ with relative weights $w_{i \bullet}^{(k)}$ :

$\operatorname{cov}_{w_{i:}^{(k)}}\left(B^{(k)}, B^{(l)}\right)=\frac{1}{I-1} \sum_{i=1}^{I}\left(B_{i}^{(k)}-\overline{B^{(k)}}\right) \cdot\left(B_{i}^{(l)}-\overline{B^{(l)}}\right) \cdot w_{i \bullet}^{(k)}$

and $\operatorname{cov}_{w_{i}^{(l)}}\left(B^{(k)}, B^{(l)}\right)$ is the sample covariance between variables $B(k), B(l)$ with relative weights $w_{i \bullet}^{(l)}$ :

$\operatorname{cov}_{w_{i:}^{(l)}}\left(B^{(k)}, B^{(l)}\right)=\frac{1}{I-1} \sum_{i=1}^{I}\left(B_{i}^{(k)}-\overline{B^{(k)}}\right) \cdot\left(B_{i}^{(l)}-\overline{B^{(l)}}\right) \cdot w_{i \bullet}^{(l)}$

For $k=1$ we get the sample variance (46) and the sample covariance (50) (for $i=1,2, \ldots, p$ ) from the first row of the sample covariance matrix of variables $B(1), B(2), \ldots, B(p)$ making use of the relative weights $w_{i \bullet}^{(1)}$. Similarly for $k=2$ 
we can read off the sample variance (46) and sample covariance (50) (for $I=1,2, \ldots, p$ ) from the second row of the sample covariance matrix of variables $B(1), B(2), \ldots, B(p)$ by making use of the relative weights $w_{i \bullet}^{(2)}$. Analogously we can obtain the sample variance (46) and sample covariance $(50)$ for $k=3,4, \ldots, p$. From these elements we can then develop the matrix

$\mathbf{S}_{\mathbf{B}}=\left(\begin{array}{cccc}s_{B^{(1)}, w_{i}^{(1)}}^{2} & \operatorname{cov}_{w_{i p}^{(1)}}\left(B^{(1)}, B^{(2)}\right) & \ldots & \operatorname{cov}_{w_{i}^{(1)}}\left(B^{(1)}, B^{(p)}\right) \\ \operatorname{cov}_{w_{i}^{(2)}}\left(B^{(2)}, B^{(1)}\right) & s_{B^{(2)}, w_{i}^{(2)}}^{2} & \ldots & \operatorname{cov}_{w_{i}^{(2)}}\left(B^{(2)}, B^{(p)}\right) \\ \vdots & \vdots & \ddots & \vdots \\ \operatorname{cov}_{w_{i}^{(p)}}\left(B^{(p)}, B^{(1)}\right) & \operatorname{cov}_{w_{i}^{(p)}}\left(B^{(p)}, B^{(2)}\right) & \ldots & s_{B^{(p)}, w_{i}^{(p)}}^{2}\end{array}\right)$

whose $k^{\text {th }}$ row is the $k^{\text {th }}$ row of the sample covariance matrix of variables $B(1), B(2), \ldots, B(p)$ making use of the relative weights $w_{i \bullet}^{(k)}$..

If we look at formula (48) and also formula (49) we see that the estimates of the nondiagonal elements of the kth row of the matrix T are a multiple $\quad I \cdot \frac{c^{(k)}}{w_{\bullet \bullet}^{(k)}} \quad$ of the $k^{\text {th }}$ row of the matrix $\mathbf{S}_{\mathrm{B}}$. From formula (47) it is obvious that the estimate of the $k^{\text {th }}$ diagonal element of the matrix $\mathbf{T}$ is a multiple $I \cdot \frac{c^{(k)}}{w_{\bullet \bullet}^{(k)}}$ of the difference between the $k^{\text {th }}$ diagonal element of the matrix $S_{B}$ and the $k^{\text {th }}$ diagonal element of the matrix $\hat{\mathbf{S}}$. Given that the matrix $\hat{\mathbf{S}}$ is diagonal with elements $\widehat{\sigma_{k}^{2}}(k=1,2, \ldots, p)$ we can define the matrix

$$
\mathbf{R}=\left(\mathbf{S}_{\mathbf{B}}-\hat{\mathbf{S}}\right) \otimes \mathbf{C}
$$

where $\otimes$ is the Hadamard matrix product and matrix $\mathbf{C}$ of type $p \times p$ has elements

$$
\mathbf{C}=I \cdot\left(\begin{array}{c}
\frac{c^{(1)}}{w_{\bullet}^{(1)}} \\
\vdots \\
\frac{c^{(p)}}{w_{\bullet \bullet}^{(p)}}
\end{array}\right) \cdot\left(\begin{array}{lll}
1 & \cdots & 1
\end{array}\right)=I \cdot\left(\begin{array}{ccc}
\frac{c^{(1)}}{w_{\bullet}^{(1)}} & \cdots & \frac{c^{(1)}}{w_{\bullet \bullet}^{(1)}} \\
\vdots & \ddots & \vdots \\
\frac{c^{(p)}}{w_{\bullet \bullet}^{(p)}} & \cdots & \frac{c^{(p)}}{w_{\bullet \bullet}^{(p)}}
\end{array}\right)
$$

An estimate of the covariance matrix $\mathbf{T}$ is then the matrix

$$
\widehat{\mathbf{T}}=\frac{1}{2} \cdot\left(\mathbf{R}+\mathbf{R}^{\mathrm{T}}\right)
$$

If any of its diagonal elements are negative, then in accordance with formula (34) we replace them by the value 0 and if any of its non-diagonal elements do not meet the condition $\left|\widehat{\tau_{k l}}\right| \leq \widehat{\tau_{k}} \cdot \widehat{\tau_{l}}$, then in accordance with formula (42) we replace them by the value $\operatorname{sgn}\left(\frac{\widehat{\tau_{k l}}+\widehat{\tau_{k l}^{*}}}{2}\right) \cdot \sqrt{\widehat{\tau_{k}^{2}} \cdot \widehat{\tau_{l}^{2}}}$

\section{Example of Application}

An unnamed insurance company divides its MTPL portfolio into eight tariff classes. For this type of insurance the insurer has available not only its own data, but also summary data from other companies. Specifically for each risk class it has available the following statistics:

$\bar{X}_{i} \quad$ - the average claim amount (in €), per contract year, for a period of $\mathrm{n}$ years,

$$
\widehat{\sigma_{i}}=\sqrt{\frac{1}{n-1} \sum_{j=1}^{n}\left(X_{i j}-\overline{X_{i}}\right)^{2} w_{i j}}
$$

- the sample standard deviation of the average claim amounts for the period of $n$ years,

$w_{i}$. $\quad-$ the number of contracts for the period of $n$ years.

These data are set out in Table 1.

The actuary has the task of setting the net premium for the next insurance year. Given that one expects a significant dependence between the own company data and that of the other insurers, it is desirable to use the multidimensional credibility model to set the net premium for each tariff class. In order to get the credibility estimate of the net premium using the multidimensional model we need to carry out the calculations as shown in Table 2. 


\section{Finance}

\section{Tab. 1: Own data and data from other insurers}

\begin{tabular}{c||c|c|r||r|r|r}
\multicolumn{1}{c||}{$\begin{array}{c}\text { Risk class } \\
i\end{array}$} & \multicolumn{4}{c||}{ Own data } & \multicolumn{3}{c}{ Other insurers' data } \\
\cline { 2 - 7 } & $\overline{X_{i}}$ & $\widehat{\sigma}_{i}$ & $w_{i \bullet}$ & $\overline{X_{i}}$ & $\widehat{\sigma}_{i}$ & $w_{i \bullet}$ \\
\hline 1 & 40 & 78 & 297 & 48 & 73 & 2,893 \\
\hline 2 & 54 & 92 & 1,606 & 49 & 88 & 5,982 \\
\hline 3 & 71 & 110 & 5,232 & 73 & 120 & 18,704 \\
\hline 4 & 78 & 109 & 6,283 & 77 & 99 & 22,981 \\
\hline 5 & 79 & 146 & 5,340 & 83 & 113 & 21,056 \\
\hline 6 & 98 & 195 & 3,189 & 109 & 147 & 16,795 \\
\hline 7 & 133 & 246 & 2,576 & 116 & 181 & 8,994 \\
\hline 8 & 169 & 382 & 353 & 143 & 236 & 2,703 \\
\hline
\end{tabular}

Source: own

\section{Tab. 2: Table of calculations}

\begin{tabular}{|c|c|c|c|c|c|c|c|c|}
\hline$i$ & $B_{i}^{(1)}$ & $w_{i \bullet}^{(1)}$ & ${\widehat{\sigma_{i}^{2}}}^{(1)}$ & $\left(1-\frac{w_{i \bullet}^{(1)}}{w_{\bullet \bullet}^{(1)}}\right) \frac{w_{i \bullet}^{(1)}}{w_{\bullet \bullet}^{(1)}}$ & $B_{i}^{(2)}$ & $w_{i \bullet}^{(2)}$ & ${\widehat{\sigma_{i}^{2}}}^{(2)}$ & $\left(1-\frac{w_{i \bullet}^{(2)}}{w_{\bullet \bullet}^{(2)}}\right) \frac{w_{i \bullet}^{(2)}}{w_{\bullet \bullet}^{(2)}}$ \\
\hline 1 & 40 & 297 & 6,084 & 0.012 & 48 & 2,893 & 5,329 & 0.028 \\
\hline 2 & 54 & 1,606 & 8,464 & 0.060 & 49 & 5,982 & 7,744 & 0.056 \\
\hline 3 & 71 & 5,232 & 12,100 & 0.166 & 73 & 18,704 & 14,400 & 0.152 \\
\hline 4 & 78 & 6,283 & 11,881 & 0.189 & 77 & 22,981 & 9,801 & 0.177 \\
\hline 5 & 79 & 5,340 & 21,316 & 0.169 & 83 & 21,056 & 12,769 & 0.166 \\
\hline 6 & 98 & 3,189 & 38,025 & 0.112 & 109 & 16,795 & 21,609 & 0.140 \\
\hline 7 & 133 & 2,576 & 60,516 & 0.093 & 116 & 8,994 & 32,761 & 0.082 \\
\hline 8 & 169 & 353 & 145,924 & 0.014 & 143 & 2,703 & 55,696 & 0.026 \\
\hline $\boldsymbol{\Sigma}$ & $x$ & 24,876 & 304,310 & 0.814 & $x$ & 100,108 & 160,109 & 0.827 \\
\hline
\end{tabular}

Source: own calculation

By taking the simple arithmetic means of the sample variances ${\widehat{\sigma_{i}^{2}}}^{(1)}$ and the simple arithmetic means of the sample variances $\widehat{\sigma_{i}^{2}}{ }^{(2)}$ we obtain estimates of the diagonal elements of the matrix $\mathbf{S}$

$\widehat{\sigma_{1}^{2}}=\frac{1}{8} \cdot 304310=38038.75 \quad \widehat{\sigma_{2}^{2}}=\frac{1}{8} \cdot 160109=20013.63$

An estimate of the matrix $\mathbf{S}$ is therefore the matrix

$$
\hat{\mathbf{S}}=\left(\begin{array}{rrr}
38 & 038.75 & 0 \\
0 & 20013.63
\end{array}\right)
$$

To avoid the relatively lengthy calculations to estimate matrix $\mathbf{T}$ we will use the adjustments which we made earlier in the article (expressions
(45) to (52)). To calculate the elements of matrix (52) we can use several of statistical software applications. To estimate matrix $\mathbf{T}$ in this article we use correlation analysis procedure in SAS Enterprise Guide application.

First we estimate the covariance matrix of variables $B^{(1)}$ a $B^{(2)}$ (the values are shown in Tables 1 and 2), whereby we make use of the relative weights $w_{i \bullet}^{(1)}$. Similarly we estimate the covariance matrix of variables $B(1)$ a $B(2)$ making use of the relative weights $w_{i}^{(2)}$. We thus get the covariance matrices shown in Table 3. 


\begin{tabular}{|c|c|}
\hline 2 Variables: & B1 $B 2$ \\
\hline Weight Variable: & w1 \\
\hline
\end{tabular}

\begin{tabular}{|c|c|c|}
\hline \multicolumn{3}{|c|}{ Covariance Matrix. DF = 7 } \\
\hline & B1 & B2 \\
\hline B1 & $1,803,234.505$ & $1,445,493.254$ \\
\hline B2 & $1,445,493.254$ & $1,307,839.123$ \\
\hline
\end{tabular}

\begin{tabular}{|c|c|}
\hline 2 Variables: & B1 $\mathrm{B} 2$ \\
\hline Weight Variable: & w2 \\
\hline
\end{tabular}

\begin{tabular}{|c|c|c|}
\hline \multicolumn{3}{|c|}{ Covariance Matrix. DF = 7 } \\
\hline & B1 & B2 \\
\hline B1 & $8,541,207.977$ & $6,851,228.250$ \\
\hline B2 & $6,851,228.250$ & $6,189,754.455$ \\
\hline
\end{tabular}

Source: processing in SAS Enterprise Guide

From the covariance matrix in Table 3 we develop the matrix $\mathbf{S}_{\mathbf{B}}$

$$
\mathbf{S}_{\mathbf{B}}=\left(\begin{array}{lll}
1803234.51 & 1445493.25 \\
6851228.25 & 6189754.46
\end{array}\right)
$$

From the partial results in Table 2 in accordance with formula (36) we get $c^{(1)}=\frac{7}{8} \cdot \frac{1}{0.814}=1.0746$, $c^{(2)}=\frac{7}{8} \cdot \frac{1}{0.827}=1.0583$ and in accordance with formula (54) we calculate the matrix of constants

$$
\mathbf{C}=\left(\begin{array}{ll}
0.0003456 & 0.0003456 \\
0.0000846 & 0.0000846
\end{array}\right)
$$

By means of the Hadamard product of the difference between matrices $\mathbf{S}_{\mathrm{B}}, \hat{\mathbf{S}}$ and matrix $\mathbf{C}$ we calculate the matrix

$$
\mathbf{R}=\left(\mathbf{S}_{\mathbf{B}}-\hat{\mathbf{S}}\right) \otimes \mathbf{C}=\left(\begin{array}{ll}
610.054 & 499.564 \\
579.425 & 521.790
\end{array}\right)
$$

Its diagonal elements represent estimates of the diagonal elements of the matrix $\mathbf{T}$, i.e. $\widehat{\tau_{1}^{2}}=610.054, \widehat{\tau_{2}^{2}}=521.790$. By taking the simple arithmetic means of the elements $r_{12}$ a $r_{21}$ of the matrix $\mathbf{R}$ we estimate the elements $\tau_{12}$ a $\tau_{21}$ :

$$
\widehat{\tau_{12}}=\widehat{\tau_{21}}=\frac{499.564+579.425}{2}=539.495
$$

Given that the resulting estimates of the diagonal elements (variances) of the matrix $\mathbf{T}$ are positive and the product of their square roots (standard deviations) is greater than the estimate of the non-diagonal element (covariance), an estimate of the matrix $\mathbf{T}$ is given by the matrix

$$
\widehat{\mathbf{T}}=\left(\begin{array}{ll}
610.054 & 539.495 \\
539.495 & 521.790
\end{array}\right)
$$

We can use the matrices $\hat{\mathbf{S}}$ and $\hat{\mathbf{T}}$ to estimate the credibility matrix. In accordance with formulae (17) and (22) we get the credibility matrices shown in Table 4 . The credibility matrix relating to the th tariff class represents the weight for the vector of individual premiums $\mathbf{B}_{i}$. Its complement to the unit matrix represents the weight for the vector of collective premiums. In accordance with formula (24) its estimate is the vector

$$
\begin{gathered}
\hat{\hat{\boldsymbol{\mu}}}=\left(\sum_{i=1}^{I} \mathbf{Z}_{i}\right)^{-1} \cdot \sum_{i=1}^{I}\left(\mathbf{Z}_{i} \cdot \mathbf{B}_{i}\right)=\left(\begin{array}{ll}
5.642 & 2.415 \\
0.227 & 7.721
\end{array}\right)^{-1} . \\
\cdot\left(\begin{array}{l}
713.329 \\
694.722
\end{array}\right)=\left(\begin{array}{l}
89.033 \\
87.355
\end{array}\right)
\end{gathered}
$$

Using the described approach of weighting the vector of individual premiums and estimating the vector of collective premiums in accordance with formula (16) we get the homogeneous credibility estimates of the vector of net premiums for each tariff class. These estimates are shown in the last column of Table 4. 


\section{Tab. 4: The credibility matrices and the net premium vectors}

\begin{tabular}{|c|c|c|c|c|c|}
\hline \multirow[b]{2}{*}{$i$} & \multirow{2}{*}{\multicolumn{2}{|c|}{$\begin{array}{c}\text { Credibility matrices } \\
\qquad \mathbf{Z}_{i}\end{array}$}} & \multicolumn{3}{|c|}{ Net premium vectors } \\
\hline & & & $\begin{array}{c}\text { individual } \\
\mathbf{B}_{i}\end{array}$ & $\begin{array}{c}\text { collective } \\
\hat{\hat{\mu}}\end{array}$ & $\begin{array}{l}{\widehat{\widehat{\mu\left(\theta_{i}\right)}}}^{\text {credibility }} \\
\text { hom }\end{array}$ \\
\hline \multirow{2}{*}{1} & $(0.317$ & 0.697 & $(40)$ & $(89.033)$ & $(46.058)$ \\
\hline & 0.038 & 0.949 & $(48)$ & $(87.355)$ & 48.181 \\
\hline \multirow{2}{*}{2} & $(0.702$ & $0.306)$ & $(54)$ & $(89.033)$ & 52.698 \\
\hline & 0.043 & $0.949)$ & $(49)$ & $(87.355)$ & 49.433 \\
\hline \multirow{2}{*}{3} & $(0.880$ & $0.124)$ & $(71)$ & $(89.033)$ & $(71.386)$ \\
\hline & 0.018 & $0.979)$ & $(73)$ & $(87.355)$ & 72.971) \\
\hline \multirow{2}{*}{4} & $(0.898$ & 0.105 & $(78)$ & $(89.033)$ & 78.035 \\
\hline & 0.015 & $0.983)$ & $(77)$ & $(87.355)$ & 77.012 \\
\hline \multirow{2}{*}{5} & $(0.882$ & $0.122)$ & $(79)$ & $(89.033)$ & $(79.653)$ \\
\hline & 0.016 & $0.981)$ & $(83)$ & $(87.355)$ & 82.918 \\
\hline \multirow{2}{*}{6} & $(0.818$ & 0.188 & $(98)$ & $(89.033)$ & $(100.435)$ \\
\hline & $0.01 \mathrm{~S}$ & $0.978)$ & (109) & $(87.355)$ & 108.700 \\
\hline \multirow{2}{*}{7} & $(0.787$ & $0.219)$ & $(133)$ & $(89.033)$ & $(129.919)$ \\
\hline & 0.033 & $0.962)$ & $(116)$ & $(87.355)$ & 116.356 \\
\hline \multirow{2}{*}{8} & $(0.35$ & 0.655 & $(169)$ & $(89.033)$ & $(154.078)$ \\
\hline & 0.04 & 0.940 & $(143)$ & $(87.355)$ & 143.267 \\
\hline
\end{tabular}

Source: own calculation

Understandably of priority interest to the insurer are the net premium rates for the next insurance year in respect of the individual risk classes in its own portfolio. These rates are the first elements of the vectors $\widehat{\widehat{\boldsymbol{\mu}\left(\theta_{i}\right)}}=\left(\mu_{1}\left(\theta_{i}\right)^{\text {hom }} \mu_{2}\left(\theta_{i}\right)^{\text {hom }}\right)^{\mathrm{T}}$ for $i=1,2, \ldots, 8$, which are shown in the last column of Table 4 . The given credibility net premium for a particular risk class takes into account not only data relating to the claims experience of that class for the foregoing years and the past data for similar risk classes, which together form the insurer's own portfolio, but also data for similar risk classes as well as the whole portfolio of other insurers.

Although we cannot interpret the credibility matrix as simply as we could the credibility factors in the one-dimensional credibility model, we can say that the insurer when setting its premiums can the least rely on its own data for the particular risk classes in the case of the first $(i=1)$ and last $(i=8)$ tariff classes, for which the first diagonal elements $\left(z_{11}=0.317\right.$ and $\left.z_{11}=0.358\right)$ of the credibility matrix $\mathbf{Z}_{\mathrm{i}}$ are the smallest. These elements of the matrix $\mathbf{Z}_{i}$ reflect the fact that the first and last tariff classes of the insurer possess the least amount of historical data $\left(w_{10}^{(1)}=297\right.$ and $\left.w_{8 .}^{(1)}=353\right)$, and therefore greater reliance has to be placed on the data for the insurer's other classes of contract or on other insurers' data.

On the other hand when setting the premium the most reliable own data $\left(z_{11}=0.898\right)$ relate to the fourth tariff class, where the insurer has the most data $\left(w_{4 \bullet}^{(1)}=6283\right)$. For this reason the difference between the credibility premium for the fourth risk class $\widehat{\widehat{\mu_{1}\left(\theta_{4}\right)}}=78.035$ and the corresponding individual premium $B_{4}^{(1)}=78$ is negligible. On the other hand for the first and last tariff classes the difference between the credibility and individual premium is not negligible. In the case of the first risk class the 
credibility premium is some $€ 6$ higher than the individual premium and in the case of the last classes some $€ 15$ lower. A graphical com- parison of the individual, collective and credibility premiums for each of the risk classes of the insurer's portfolio is shown in Figure 1.

\section{Fig. 1: A comparison of the individual, collective and credibility premiums for each of the risk classes of the insurer's portfolio}

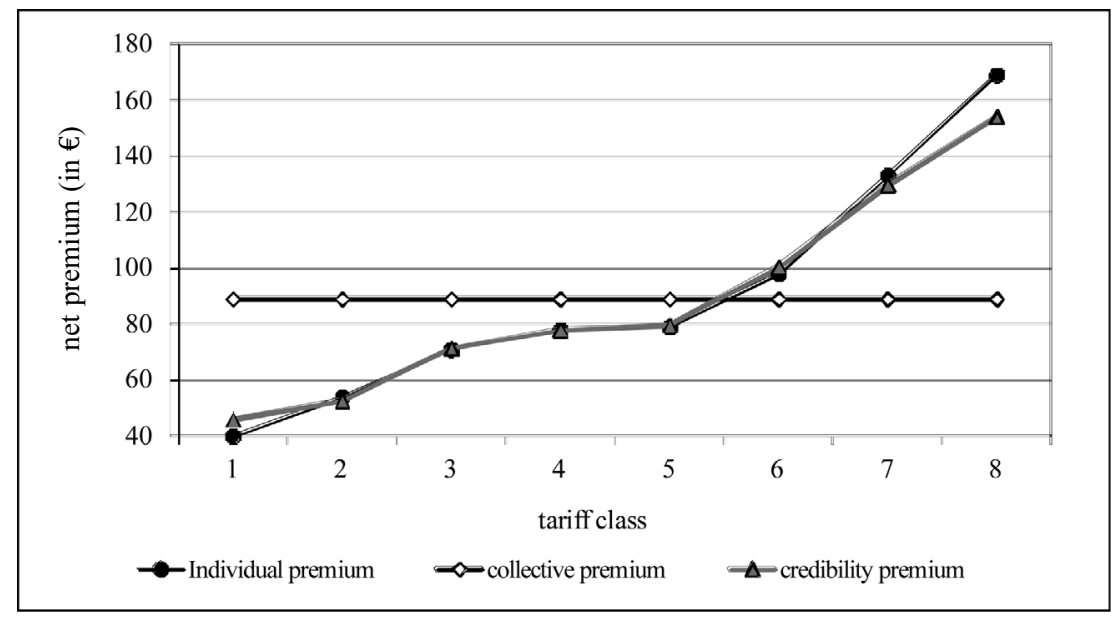

Source: own calculation and processing in Microsoft Excel

An important property of the credibility premium is the balance property. In order to verify it we first rewrite formula (25) as follows

$$
\sum_{i=1}^{I}\left[{\widehat{\widehat{\mu_{k}\left(\theta_{i}\right.}}}^{\text {hom }} \cdot \frac{w_{i \bullet}^{(k)}}{w_{\bullet \bullet}^{(k)}}\right]=\sum_{i=1}^{I}\left[B_{i}^{(k)} \cdot \frac{w_{i \bullet}^{(k)}}{w_{\bullet \bullet}^{(k)}}\right]
$$

On the right-hand side of the formula we have the average annual claim amount per contract in the portfolio for the whole $n$ year period. In the case of our example portfolio $\overline{B^{(1)}}=€ 84.290$ and for the other insurers $\overline{B^{(2)}}=€ 85.658$. The sum on the left-hand side is calculated separately for the own data and the other insurers' data in Table 5.

From Table 5 it is clear that in the portfolio with eight tariff classes the average annual claim amount per contract over the past $n$ years is equal to the average credibility premium (if the number of contracts in each tariff class is unchanged). For the own portfolio the average value is on the level of $€ 84.290$ and for the other insurers' portfolio on the level of $€$ 85.658.

The aim of the application of the multidimensional Bühlmann-Straub credibility model was to show how it is possible in determining the net premium for the individual risk class of the heterogeneous risk portfolio use three different sources of information from the previous insurance period: data from the relevant risk class, information about the claims record in other risk classes of the own portfolio and information about the claims record in risk classes of others insurance companies. As mentioned above (without proof and with reference to the source), the quadratic loss of the credibility premium in all tariff classes is smaller than the quadratic loss of the collective premium, as well as the quadratic loss of the individual premium. Therefore credibility premium provides a better estimate of the future costs of insurance losses as individual or collective estimate. Moreover, this estimate satisfies the balance property. However, it must be said, that in case of the large number of insurance contracts the 


\section{Tab. 5: Calculation table for verifying the balance property}

\begin{tabular}{|c|c|c|c|c|c|c|}
\hline \multirow[b]{2}{*}{$i$} & \multicolumn{3}{|c|}{ Own data } & \multicolumn{3}{|c|}{ Other insurers' data } \\
\hline & ${\widehat{\widehat{\mu_{1}\left(\theta_{i}\right)}}}^{\text {hom }}$ & $w_{i \bullet}^{(1)}$ & $\widehat{\widehat{\mu_{1}\left(\theta_{i}\right)}}$ hom $\cdot \frac{w_{i \bullet}^{(1)}}{w_{\bullet \bullet}^{(1)}}$ & ${\widehat{\widehat{\mu_{2}\left(\theta_{i}\right)}}}^{\text {hom }}$ & $w_{i \bullet}^{(2)}$ & $\widehat{\widehat{\mu_{2}\left(\theta_{i}\right)}}$ hom $\cdot \frac{w_{i \bullet}^{(2)}}{w_{\bullet \bullet}^{(2)}}$ \\
\hline 1 & 46.058 & 297 & 0.550 & 48.181 & 2,893 & 1.392 \\
\hline 2 & 52.698 & 1,606 & 3.402 & 49.433 & 5,982 & 2.954 \\
\hline 3 & 71.386 & 5,232 & 15.014 & 72.971 & 18,704 & 13.634 \\
\hline 4 & 78.035 & 6,283 & 19.710 & 77.012 & 22,981 & 17.679 \\
\hline 5 & 79.653 & 5,340 & 17.099 & 82.918 & 21,056 & 17.440 \\
\hline 6 & 100.435 & 3,189 & 12.875 & 108.700 & 16,795 & 18.236 \\
\hline 7 & 129.919 & 2,576 & 13.454 & 116.356 & 8,994 & 10.454 \\
\hline 8 & 154.078 & 353 & 2.186 & 143.267 & 2,703 & 3.868 \\
\hline$\Sigma$ & $x$ & 24,876 & 84.290 & $x$ & 100,108 & 85.658 \\
\hline
\end{tabular}

Source: own calculation

differences between individual and credible premiums are negligible, as shown by the mentioned application in case of risk classes 3 to 6 .

\section{Conclusion}

Credibility theory belongs amongst the socalled "experience rating" techniques and serves for calculating and continuously updating the premium. Similar to Bayesian estimates, the estimates gained from credibility models also emerge from the a priori data from a portfolio of contracts and from individual data. As compared with Bayesian estimates the credibility estimates of the premium are relatively straightforward, as they do not require the estimation of the whole a priori distribution, but only its first two moments. Further the credibility premiums are easy to interpret. Thanks to this credibility theory has many possible applications in actuarial work.

Without doubt the most important model in modern credibility theory is the BühlmannStraub model which can have wide applications and represents a base for further more specific models. One such model is the multidimensional model, to which we have devoted this paper. In the paper we have mentioned the generalisation of the onedimensional Bühlmann-Straub credibility model to the multidimensional credibility model. Given its use is mainly in non-life insurance and a significant proportion of the written premiums in non-life insurance relate to MTPL we have applied the multidimensional credibility model to a portfolio of such contracts. In order to minimise the calculation overhead inherent in the multidimensional credibility model we have adjusted the estimates of the structural parameters so that we can use the methods and approaches of statistical induction. Thanks to these adjustments we have been able to use in our example application the procedures in the SAS Enterprise Guide statistical software. From the example application it ensues amongst other things that the actuary should reach for the methods of credibility theory in particular where there is a relatively small amount of data relating to the insured risk. At the current time, when non-life insurers, particularly with respect to MPTL, are introducing new tariff factors and the degree of portfolio segmentation is increasing, a lack of statistical data for assessing risk is quite common. But not even several years' experience with a given tariff class are necessarily sufficient to value the insurance risk. Insurance mathematicians should therefore not ignore the data from the companies' own similar contracts or data from other sources (e.g. from other insurers). Credibility models are the tool that can be used with such data sources when setting the net premium. Credibility models permit the setting of a credibility premium, which in the observed 
past period of $n$ years (on the assumption of an unchanged number of contracts in each tariff class) would cover the total claim payments for the whole portfolio. Thanks also to this socalled balance property the credibility premium meet the conditions for use in insurance practice.

The paper is written in the framework of the projects VEGA 1/0806/14 SCR calculation to cover the risks of non-life insurance in accordance with practical needs and project GA ČR 402/09/1866 Modelling, simulation and management of insurance risks.

\section{References}

[1] BÜHLMANN, H., GISLER, A. A Course in Credibility Theory and its Applications. Berlin: Springer, 2005. ISBN 3540257535.

[2] BÜHLMANN, H., GISLER, A., KOLLÖFFEL, D. Multidimensional Credibility applied to estimating the frequency of big claims. ASTIN Bulletin [online]. ETH Zürich and Winterthur Insurance Company, 2003. [cit. 2010-10-22]. 34 p. (PDF). Available from: http://www.actuaries.org/ASTIN/ Colloquia/Berlin/Buhlmann_Gisler_Kolloffel.pdf.

[3] BÜHLMANN, H., STRAUB, E. Glaubwürdigkeit für Schadensätze. Mitteilungen der Vereinigung Schweizerischer Versicherungs-mathematiker. 1970, Vol. 70, pp. 111-133.

[4] DANNENBURG, D.R. Basic actuarial credibility models - Evaluations and extensions. Amsterdam, 1996. Dissertation thesis (PhD.). Tinbergen Institute.

[5] GOOVAERTS, M.J., KAAS, R., VAN HEERWAARDEN, A.E., Bauwelinck, T. Effective
Actuarial Methods. Amsterdam: North-Holland, 1990. ISBN 978-0444883995.

[6] HERZOG, T.N. Introduction to credibility theory. 3rd ed. Winsted: ACTEX Publications, 1999. ISBN 978-1566983747.

[7] KAAS, R., GOOVAERTS, M. J., DHAENE, J., DENUIT, M. Modern actuarial risk theory. Boston: Kluwer academic publishers, 2001. ISBN 1-40202952-7.

[8] PACÁKOVÁ, V. Aplikovaná poistná štatistika. Bratislava: IURA Edition, 2004. ISBN 80-8078-004-8. [9] ŠOLTÉS, E. Modely kredibility na výpočet poistného. Bratislava: Vydavatel'stvo Ekonóm, 2009. ISBN 978-80-225-2798-9.

prof. RNDr. Viera Pacáková, PhD. University of Pardubice Faculty of Economics and Administration Institute of Mathematics and Quantitative Methods viera.pacakova@upce.cz

doc. Mgr. Erik Šoltés, PhD. University of Economics in Bratislava Faculty of Economic Informatics Department of Statistics erik.soltes@euba.sk

doc. RNDr. Bohdan Linda, PhD. University of Pardubice

Faculty of Economics and Administration Institute of Mathematics and Quantitative Methods bohdan.linda@upce.cz 


\section{Abstract}

\section{MULTIDIMENSIONAL CREDIBILITY MODEL AND ITS APPLICATION}

\section{Viera Pacáková, Erik Šoltés, Bohdan Linda}

Solvency II project places emphasis on the modelling and management of risks of the insurance companies. This requires further improvement in actuarial methods and their application in insurance practice. Improving the quality of premium calculation methods is an effective factor in reducing the insurance technical risk of an insurer. Presentation the methods of premium calculation and its permanent updating is the aim of this article.

Credibility theory is an experience rating technique to determine premiums, claim frequencies or claim sizes. Credibility models are based on the realistic concept of a heterogeneous insurance portfolio. Therefore, two sources of information are used in the calculation of the credibility estimators for the individual risk: typically little knowledge about the individual risk and quite extensive statistical information about entire portfolio. The most important model in the credibility theory is Bühlmann-Straub model. This model has a wide range of possibilities to be used in praxis mainly in general insurance. Besides that this model is a basis for other more specific models such as hierarchical, multidimensional or regression credibility models. In this article we deal with generalisation of one-dimensional Bühlmann-Straub credibility model to the multidimensional credibility model. We mainly focus on estimation of so-called structural parameters and usage of SAS Enterprise Guide application when estimating. The multidimensional Bühlmann-Straub credibility model is applied based the real data in motor vehicle third party liability insurance.

Key Words: Credibility premium, Bühlmann-Straub model, multidimensional credibility model, estimation of structural parameters, motor vehicle third party liability insurance.

JEL Classification: C11, C13, G22.

DOI: 10.15240/tul/001/2014-2-013 